CLINICAL STUDY

\title{
Mitotane levels predict the outcome of patients with adrenocortical carcinoma treated adjuvantly following radical resection
}

\author{
M Terzolo, A E Baudin ${ }^{1}$, A Ardito, M Kroiss ${ }^{2}$, S Leboulleux ${ }^{1}$, F Daffara, P Perotti, R A Feelders ${ }^{3}$, J H deVries ${ }^{4}$, \\ B Zaggia, S De Francia ${ }^{5}$, M Volante ${ }^{6}$, H R Haak ${ }^{7}$, B Allolio ${ }^{2}$, A Al Ghuzlan ${ }^{1}$, M Fassnacht ${ }^{2,8}$ and A Berruti $^{9}$ \\ Internal Medicine I, Department of Clinical and Biological Sciences, San Luigi Gonzaga Hospital, University of Turin, Regione Gonzole 10, 10043 \\ Orbassano, Italy, ${ }^{1}$ Endocrine Oncology and Nuclear Medicine, IGR, Villejuif, France, ${ }^{2}$ Department of Medicine I, University Hospital of Würzburg, \\ Würzburg, Germany, ${ }^{3}$ Erasmus Medical Centre, Rotterdam, The Netherlands, ${ }^{4}$ Academic Medical Centre, Amsterdam, The Netherlands, ${ }^{5}$ Pharmacology \\ and ${ }^{6}$ Pathology, San Luigi Gonzaga Hospital, University of Turin, Orbassano, Italy, ${ }^{7}$ Internal Medicine, Maxima Medical Centre, Eindhoven, The \\ Netherlands, ${ }^{8}$ Department of Internal Medicine IV, Hospital of the University of Munich, Munich, Germany and ${ }^{9}$ Oncology, San Luigi Gonzaga Hospital, \\ University of Turin, Orbassano, Italy
}

(Correspondence should be addressed to A Ardito; Email: arianna.ardito@gmail.com)

\begin{abstract}
Context: Mitotane plasma concentrations $\geq 14 \mathrm{mg} / \mathrm{l}$ have been shown to predict tumor response and better survival in patients with advanced adrenocortical carcinoma (ACC). A correlation between mitotane concentrations and patient outcome has not been demonstrated in an adjuvant setting. Objective: To compare recurrence-free survival (RFS) in patients who reached and maintained mitotane concentrations $\geq 14 \mathrm{mg} / \mathrm{l}$ vs patients who did not.

Design and setting: Retrospective analysis at six referral European centers.

Patients: Patients with ACC who were radically resected between 1995 and 2009 and were treated adjuvantly with mitotane targeting concentrations of $14-20 \mathrm{mg} / \mathrm{l}$.

Main outcome measures: RFS (primary) and overall survival (secondary).

Results: Of the 122 patients included, 63 patients (52\%) reached and maintained during a median follow-up of 36 months the target mitotane concentrations (group 1) and 59 patients (48\%) did not (group 2). ACC recurrence was observed in 22 patients of group 1 (35\%) and 36 patients in group 2 $(61 \%)$. In multivariable analysis, the maintenance of target mitotane concentrations was associated with a significantly prolonged RFS (hazard ratio (HR) of recurrence: $0.418,0.22-0.79 ; P=0.007$ ), while the risk of death was not significantly altered (HR: $0.59,0.26-1.34 ; P=0.20$ ). Grades 3-4 toxicity was observed in 11 patients $(9 \%)$ and was managed with temporary mitotane discontinuation. None of the patients discontinued mitotane definitively for toxicity.

Conclusions: Mitotane concentrations $\geq 14 \mathrm{mg} / \mathrm{l}$ predict response to adjuvant treatment being associated with a prolonged RFS. A monitored adjuvant mitotane treatment may benefit patients after radical removal of ACC.
\end{abstract}

European Journal of Endocrinology 169 263-270

\section{Introduction}

Adrenocortical carcinoma (ACC) is a rare and aggressive endocrine tumor showing an annual incidence of $0.5-2$ cases/million population $(1,2)$. Surgery is the mainstay of therapy and complete surgical removal of ACC is the most important prognostic factor for longterm survival together with stage. Recurrence after apparently complete resection is frequent and may be found in up to $70-85 \%$ of patients with high proliferation index or locally advanced disease $(3,4,5$, 6,7 ), an observation suggesting that adjuvant treatment concepts have a major role in ACC management.

The adjuvant use of mitotane (o, $\mathrm{p}^{\prime}$-DDD), an adrenolytic drug used for treating advanced ACC since the $1960 \mathrm{~s}(3,4,8)$, has become increasingly popular after the demonstration that post-operative mitotane treatment was associated with a significantly reduced risk of recurrence and death in a large series of patients with radically operated ACC $(9,10)$. Given its retrospective nature, however, this study could not provide a definitive demonstration of the efficacy of mitotane in the adjuvant setting $(11,12)$.

For most experts, monitoring of plasma mitotane levels is currently part of the management of ACC patients treated with mitotane, because the drug is known to have a narrow therapeutic window (13). Indeed, mitotane plasma levels have been shown to be associated with both therapeutic efficacy and drugrelated toxicity in advanced ACC $(14,15)$ and a recent 
study has confirmed that a mitotane concentration above $14 \mathrm{mg} / \mathrm{l}$ is a predictor of tumor response and better survival (16). A similar strategy of targeting a threshold of $14 \mathrm{mg} / \mathrm{l}$ has been advocated in the adjuvant setting, despite the fact that it is presently unknown if the relationship between plasma concentrations of mitotane and its efficacy is applicable also to patients treated adjuvantly without evidence of disease $(13,17)$.

The aim of the present retrospective multicenter study was to evaluate whether mitotane plasma concentrations are associated with the outcome of patients receiving adjuvant mitotane treatment following radical resection of ACC. We assessed whether the currently used threshold of $14 \mathrm{mg} / \mathrm{l}$ may predict a beneficial effect of the drug on recurrence-free survival (RFS).

\section{Subjects and methods}

\section{Patients}

We performed a retrospective analysis among patients with ACC who had undergone radical surgery between January 1995 and December 2009 at six tertiary referral centers in Europe (San Luigi Gonzaga Hospital, Orbassano, Italy; Institute Gustave Roussy, Villejuif, France; University Hospital of Würzburg, Würzburg, Germany; Erasmus Medical Centre, Rotterdam, The Netherlands; Academic Medical Centre, Amsterdam, The Netherlands; and Maxima Medical Center, Eindhoven, The Netherlands) participating in the European Network for the Study of Adrenal Tumors (ENS@T; www.ensat.org). All patients who had undergone radical resection and monitored adjuvant mitotane treatment were included in the study. Follow-up for this study was closed in December 2010. During the study period, adjuvant mitotane was recommended in patients at perceived high risk of recurrence in all centers but two. At Orbassano, the policy was to recommend adjuvant mitotane treatment to all patients following resection of ACC, while at Würzburg the policy was until 2006 to offer mitotane only to "high risk patients', and in 2006-2009 to all patients.

To be eligible to this study the patients had to meet the following inclusion criteria: age of 16 years or older, histologically confirmed diagnosis of ACC, complete tumor resection, availability of postoperative follow-up information including results of imaging tests (computed tomography (CT) or magnetic resonance imaging scans), and regular determinations of plasma mitotane concentration. Exclusion criteria were macroscopically incomplete resection, incomplete tumor staging, concomitant cancers within the previous 5 years except for nonmelanoma skin cancer radically treated, clinically significant concomitant disease, incomplete follow-up information or follow-up duration of $<6$ months, initiation of mitotane treatment longer than 6 months after surgery, and concomitant postoperative adjuvant therapies (chemotherapy or radiotherapy).

All data were obtained by reviewing patient history, discharge summaries, medical records, and source documents. Data were retrieved by trained medical personnel using specifically tailored data forms. We collected data on the basis of clinical and demographical characteristics, the date of diagnosis, the stage at diagnosis, the hormonal work-up, the date and type of surgery, the pathology report, the date of recurrence, and either the date and cause of death or the date of the last follow-up visit. The following information concerning mitotane treatment was collected: treatment dose and duration, adverse effects, reasons for discontinuation, and results of monitoring of plasma concentrations. The Institutional Ethics Committee at each clinical center approved the study except that in The Netherlands where retrospective use of anonymously treated data does not need to be approved by Ethics Committees (http://www.ccmo.org). All patients provided written informed consent.

R0 resection was defined as no evidence of macroscopic residual disease on the basis of surgical reports, histopathological analysis, and postoperative imaging. All diagnoses were confirmed by experienced pathologists according to the Weiss criteria with a score of 3 , or more, identifying malignancy $(18,19)$. Staging at diagnosis was based on imaging studies and was corroborated by the findings at surgery. Staging was reported according to the ENS@T staging system (20). Disease recurrence was defined as radiologic evidence of a new tumor lesion during follow-up.

\section{Study protocol}

During the study period, monitored mitotane treatment aimed at reaching and maintaining a target plasma concentration of $14-20 \mathrm{mg} / \mathrm{l}(13,17)$ was a standard of care following removal of ACC in the participating centers. In the absence of intolerability to mitotane, adjuvant treatment was scheduled for at least 2 years, or until ACC recurrence.

All patients received the same mitotane formulation (Lysodren, $500 \mathrm{mg}$ tablets) that was purchased by Bristol-Meyers Squibb (New York, NY, USA), until 2003 and thereafter by Laboratoire HRA Pharma (Paris, France). Mitotane was given orally following a schedule of progressive dose increments according to local protocols and patient compliance aiming to reach concentrations between 14 and $20 \mathrm{mg} / \mathrm{l}(14,15)$. When such or even higher concentrations were attained, doses were tapered with further individual dose adjustments guided by the results of mitotane measurement and toxicity assessment. In the event of unacceptable side effects, the patients were allowed to return to a lower dose or discontinue mitotane temporarily, restarting with a lower dose. All patients received glucocorticoid replacement and dosing of 
Table 1 Baseline characteristics of the patients.

\begin{tabular}{lc}
\hline Age (years) & \\
Median (range) & $45(16-76)$ \\
Sex $(n(\%))$ & $53(43.4)$ \\
Male & $69(56.6)$ \\
Female & $10(8.2)$ \\
Tumor stage $(n(\%))$ & $79(64.8)$ \\
I & $33(27.0)$ \\
II & \\
III & $10.7(3.0-25.0)$ \\
Tumor size (cm) & \\
Median (range) & $65(53.3)$ \\
Functional status $(n(\%))$ & $51(41.8)$ \\
Secreting tumor & $6(4.9)$ \\
Nonsecreting tumor & $6(2-9)^{\mathrm{a}}$ \\
Missing & \\
Weiss score & $71(58.2)$ \\
Median (range) & $42(34.4)$ \\
Mitotic index $(n(\%))$ & $9(7.4)$ \\
High & \\
Low & \\
Missing & \\
\hline
\end{tabular}

${ }^{\text {a } A}$ single patient with Weiss 2 ACC recurred after 6 months.

bigh mitotic index is a mitotic count $\geq 9 / 50 \mathrm{HPF}$; low mitotic index is a mitotic count $<9 / 50$ HPF.

steroid replacement was adjusted on the basis of clinical and biochemical evaluation. At Orbassano, 50$75 \mathrm{mg} /$ daily cortisone acetate was used in most patients, while at the other centers $30-50 \mathrm{mg} /$ daily hydrocortisone was used. Thyroxine and sex steroid hormone replacement was given if deemed appropriate (13, 17). Testosterone replacement was done using mainly testosterone enanthate.

Follow-up protocols were similar in the centers including imaging of both chest and abdomen at baseline (before introducing mitotane) and thereafter every 3-6 months until disease progression or end of the study period. At each visit, the patients underwent physical examination, routine laboratory evaluation, monitoring of mitotane concentrations, and hormonal work-up. Mitotane-related toxicity was graded using NCI CTCAE criteria version 3.0 (21).

\section{Outcomes}

The primary aim of our study was to compare RFS in patients who received adjuvant mitotane therapy after radical resection and maintained the target plasma concentrations with that of patients who did not. For the purpose of this analysis, we arbitrarily defined two groups: i) patients who reached a mitotane plasma concentration of $14 \mathrm{mg} / \mathrm{l}$, or greater, and maintained this target level in at least $75 \%$ of all measurements thereafter; ii) patients who neither reached a plasma level of $14 \mathrm{mg} / \mathrm{l}$ nor could not maintain this level. Secondary outcome measures were overall survival (OS) and adverse events associated with target mitotane concentrations.

RFS was measured from the date of surgery to the date of recurrence or death, whatever event occurred first; for patients who did not have a relapse or died, the data were censored at the date of the last follow-up visit. OS was measured from the date of surgery to the date of death, and the data were censored at the date of the last follow-up visit.

\section{Statistical analysis}

Database management and all statistical analyses were performed by using the 'Statistica' for Windows software package (StatSoft, Inc., Tulsa, OK, USA). Rates and proportions were calculated for categorical data, and median and ranges for continuous data. Normality of data was assessed by the Wilk-Shapiro's test and as data were not normally distributed, the two-tailed MannWhitney $U$ test was used to assess differences in continuous variables. Differences between categorized variables were analyzed by the $\chi^{2}$ test. Survival curves were computed according to the Kaplan-Meier method and were compared by means of the log-rank test. A Cox proportional-hazards regression analysis was used to assess univariable and multivariable analyses predicting the role of maintenance of plasma mitotane concentrations of $14 \mathrm{mg} / \mathrm{l}$ or higher. Independent variables included in the multivariable analysis were the time elapsed to achieve those concentrations, sex (male vs

Table 2 Baseline characteristics of the patients treated according to two different mitotane schedules.

\begin{tabular}{|c|c|c|}
\hline & $\begin{array}{c}\text { Low-dose } \\
\text { start-up } \\
\text { regimen }(n=77)\end{array}$ & $\begin{array}{l}\text { High-dose } \\
\text { start-up } \\
\text { regimen }(n=45)\end{array}$ \\
\hline \multicolumn{3}{|l|}{ Age (years) } \\
\hline Median (range) & $44(16-71)$ & $46(22-76)$ \\
\hline \multicolumn{3}{|l|}{$\operatorname{Sex}(n(\%))$} \\
\hline Male & $35(45.5)$ & $18(40.0)$ \\
\hline Female & $42(54.5)$ & $27(60.0)$ \\
\hline \multicolumn{3}{|l|}{ Tumor stage } \\
\hline I & $6(7.8)$ & $4(9.0)$ \\
\hline II & $53(68.8)$ & $26(58.0)$ \\
\hline III & $18(23.4)$ & $15(33.0)$ \\
\hline \multicolumn{3}{|l|}{ Tumor size $(\mathrm{cm})$} \\
\hline Median (range) & $11(3.0-25.0)$ & $9.7(3.0-21.0)$ \\
\hline \multicolumn{3}{|l|}{ Mitotic index $(n(\%))^{a}$} \\
\hline High & $48(62.3)$ & $23(51.1)$ \\
\hline Low & $28(36.4)$ & $14(31.1)$ \\
\hline Missing & $1(1.3)$ & $8(17.8)$ \\
\hline \multicolumn{3}{|l|}{ Weiss score } \\
\hline Median (range) & $6(2-9)$ & $6(3-9)$ \\
\hline \multicolumn{3}{|c|}{ Functional status $(n(\%))$} \\
\hline Secreting tumor & $43(55.8)$ & $22(48.9)$ \\
\hline Nonsecreting tumor & $33(36.8)$ & $18(40.0)$ \\
\hline Missing & $1(1.3)$ & $5(1.1)$ \\
\hline \multicolumn{3}{|l|}{$\begin{array}{l}\text { Mitotane target levels } \\
\text { reached within } \\
3 \text { months }(n(\%))\end{array}$} \\
\hline Yes & $36(46.8)$ & $19(42.2)$ \\
\hline No & $41(53.2)$ & $26(57.8)$ \\
\hline
\end{tabular}

${ }^{\text {a }} \mathrm{High}$ mitotic index is a mitotic count $\geq 9 / 50 \mathrm{HPF}$; low mitotic index is a mitotic count $<9 / 50$ HPF. 


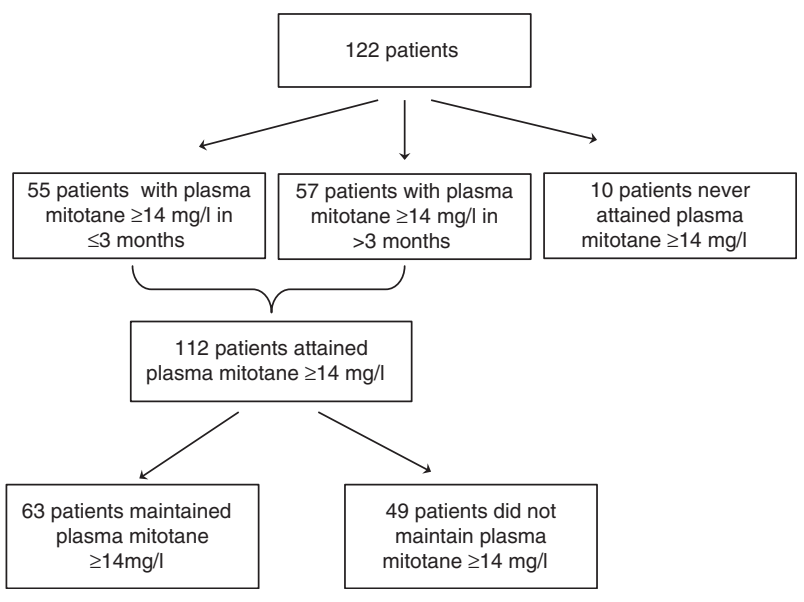

Figure 1 Study population.

female), age at diagnosis (continuous variable), ENS@T stage (I vs II vs III), hormone secretion (yes vs no), and Weiss score and mitotic index (mitosis count $\geq 9 / 50$ high power field (HPF) vs $<9 / 50 \mathrm{HPF}$ ). The mitotic index of 9/50 HPF was previously demonstrated by our group to have an independent adverse prognostic effect on either RFS or OS in a cohort of 92 patients with ACC (22). Missing data were dealt with by excluding patients from particular analyses if their files did not contain data for the required variables. All reported $P$ values are two-sided. $P$ values of $<0.05$ were considered to indicate statistical significance.

\section{Results}

One hundred and twenty-two patients entered the study. Their baseline characteristics are provided in Table 1. Mitotane was given according to a low-dose start-up regimen, defined as $<6$ g daily at the 14 th day of treatment, in 77 patients $(63.1 \%)$ while a high-dose start-up regimen, defined as more than $6 \mathrm{~g}$ daily or more, at the 14th day of treatment, was followed in 45 patients $(36.9 \%)$, according to local preferences. The two groups of patients did not differ for baseline characteristics and the percentage of patients attaining plasma mitotane levels of $14 \mathrm{mg} / \mathrm{l}$, or higher, within 3 months (Table 2). The median interval between tumor resection and initiation of mitotane was 2 months (1-4 months). The median follow-up period after surgery was 36 months (7-169 months) and the median duration of mitotane treatment was 25 months (6-165 months).

Of the 122 patients included in the study, 63 patients $(52 \%)$ maintained at least $75 \%$ of the available determinations during follow-up of the target plasma mitotane concentrations of at least $14 \mathrm{mg} / \mathrm{l}$, or higher, (group 1) and 59 patients (48\%) did not (group 2). On average, mitotane measurements were done every 3 months in 86 patients and every 6 months in
36 patients. The median follow-up duration was 40 months (11-169 months) in group 1 and 34 months (7-156 months) in group 2. A flow-chart of the patients stratified according to the plasma mitotane threshold of $14 \mathrm{mg} / \mathrm{l}$ and time needed to reach that concentration is provided in Fig. 1.

During follow-up, ACC recurrence was observed in 58 patients $(47.5 \%)$ : 22 in group $1(35.0 \%$ of the

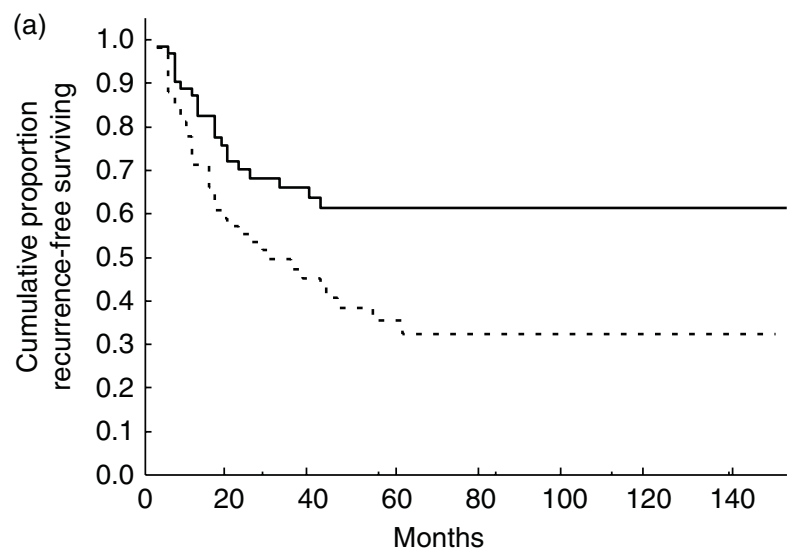

\begin{tabular}{|c|c|c|c|c|c|c|c|c|}
\hline $\begin{array}{c}\text { No. of } \\
\text { patients at risk }\end{array}$ & 0 & 20 & 40 & 60 & 80 & 100 & 120 & 140 \\
\hline Group 1 & 63 & 33 & 21 & 14 & 10 & 8 & 7 & 3 \\
\hline Group 2 & 59 & 27 & 12 & 6 & 1 & 1 & - & - \\
\hline
\end{tabular}

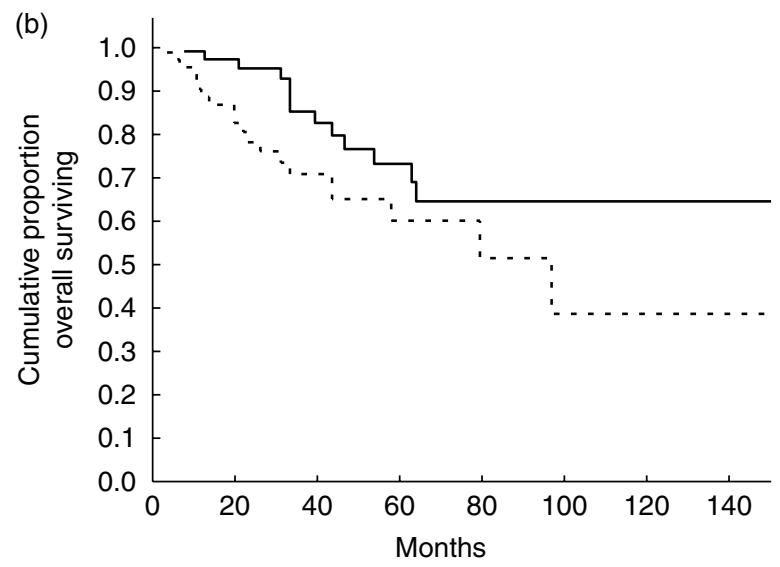

\begin{tabular}{|c|c|c|c|c|c|c|c|c|}
\hline $\begin{array}{c}\text { No. of } \\
\text { patients at risk }\end{array}$ & 0 & 20 & 40 & 60 & 80 & 100 & 120 & 140 \\
\hline Group 1 & 63 & 45 & 26 & 13 & 9 & 7 & 3 & 1 \\
\hline Group 2 & 59 & 41 & 24 & 8 & 6 & 2 & 2 & 1 \\
\hline
\end{tabular}

Figure 2 (a) Kaplan-Meier estimates for recurrence-free survival during adjuvant mitotane therapy. Solid line, patients with mitotane levels $\geq 14 \mathrm{mg} / \mathrm{l}$ during follow-up ( $n=63$ ); dashed line, patients with mitotane levels $<14 \mathrm{mg} / \mathrm{l}$ during follow-up $(n=49)$. (b) KaplanMeier estimates for overall survival during adjuvant mitotane therapy. Solid line, patients with mitotane levels $\geq 14 \mathrm{mg} / \mathrm{l}$ during follow-up $(n=63)$; dashed line, patients with mitotane levels $<14 \mathrm{mg} / \mathrm{l}$ during follow-up $(n=49)$. 


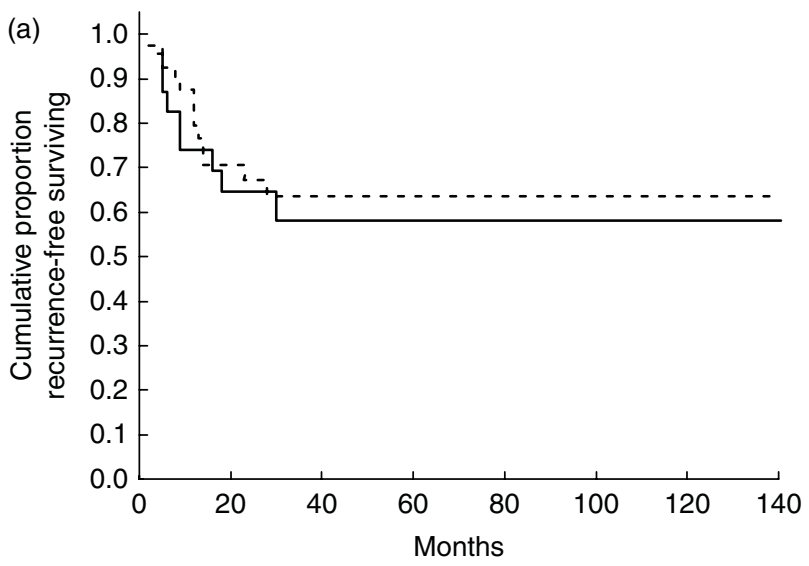

\begin{tabular}{|c|c|c|c|c|c|c|c|c|}
\hline $\begin{array}{c}\text { No. of } \\
\text { patients at risk }\end{array}$ & 0 & 20 & 40 & 60 & 80 & 100 & 120 & 140 \\
\hline Group 1 & 40 & 19 & 10 & 5 & 3 & 3 & 2 & 1 \\
\hline Group 2 & 23 & 13 & 6 & 6 & 6 & 5 & 3 & 1 \\
\hline
\end{tabular}

(b)

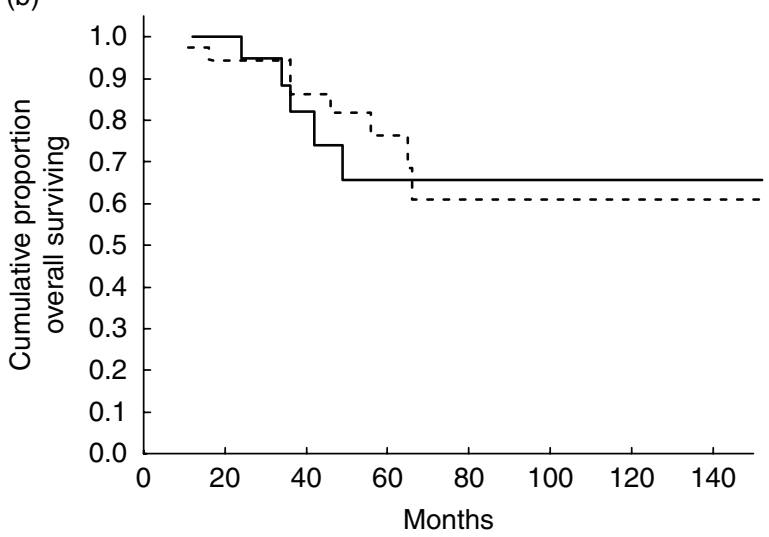

\begin{tabular}{|c|c|c|c|c|c|c|c|c|}
\hline $\begin{array}{c}\text { No. of } \\
\text { patients at risk }\end{array}$ & 0 & 20 & 40 & 60 & 80 & 100 & 120 & 140 \\
\hline Group 1 & 40 & 23 & 17 & 7 & 3 & 3 & 2 & 1 \\
\hline Group 2 & 23 & 19 & 9 & 6 & 6 & 4 & 1 & 1 \\
\hline
\end{tabular}

Figure 3 (a) Kaplan-Meier estimates for recurrence-free survival during adjuvant mitotane therapy. Dashed line, patients attaining mitotane levels $\geq 14 \mathrm{mg} / \mathrm{l}$ in $\leq 3$ month $(n=40)$; solid line, patients attaining mitotane levels $<14 \mathrm{mg} / \mathrm{l}$ in $>3$ months $(n=23)$.

(b) Kaplan-Meier estimates for overall survival during adjuvant mitotane therapy. Dashed line, patients attaining mitotane levels $\geq 14 \mathrm{mg} / \mathrm{l}$ in $\leq 3$ months $(n=40)$; solid line, patients attaining mitotane levels $<14 \mathrm{mg} / \mathrm{l}$ in $>3$ months $(n=23)$.

group) and 36 in group 2 (61.0\% of the group). Death from ACC was reported for 33 patients $(27.0 \%): 13$ in group 1 (20.6\% of the group), and 20 in group 2 (34\% of the group). At the last follow-up visit, 89 patients were alive and 59 were on mitotane treatment, 38 of whom were free of recurrence. Thirty patients had discontinued mitotane after at least 6 months of treatment by choice or following recommendation of their physicians.

In univariable analysis, the maintenance of serum mitotane concentrations of $14 \mathrm{mg} / \mathrm{l}$, or higher, was associated with longer RFS (hazard ratio (HR) of recurrence, $0.497 ; 95 \% \mathrm{CI}, 0.292-0.844 ; P=0.01$ ) and OS (HR of death, 0.511; 95\% CI, 0.253-1.029; $P=0.06)$ (Fig. 2a and b). The median RFS was not attained in group 1 and was 21 months $(95 \%$ CI, 6-36 months) in group 2. The median OS was not attained in group 1 and was 98 months (95\% CI, 50145 months) in group 2 according to the log-rank test. A rapid achievement (within 3 months) of mitotane levels of $14 \mathrm{mg} / \mathrm{l}$, or higher, did not influence either RFS or OS among the patients who maintained target mitotane concentrations (Fig. 3a and b). The results of the multivariable Cox proportional hazards models are shown in Table 3. After adjustment for sex, age at diagnosis, ENS@T stage, hormone secretion, Weiss score, and mitotic index, the patients who maintained target mitotane concentrations showed a significantly reduced risk of recurrence (adjusted HR, 0.418; 95\% CI, 0.22-0.79; $P=0.007$ ) while the risk of death was not significantly altered (adjusted HR, 0.59; 95\% CI, 0.26-1.34; $P=0.20$ ). Ad hoc analysis including time elapsed from surgery to adjuvant treatment and follow-up duration did not alter the results.

The adverse events associated with mitotane therapy are listed in Table 4. Overall, grades 3-4 toxicity was observed in 11 patients (9\%) and was managed with temporary mitotane discontinuation. None of the patients discontinued mitotane definitively for toxicity. Gastrointestinal and liver toxicities were more severe in patients treated with the high-dose start-up regimen and in this group temporary discontinuation of mitotane was more frequent.

Table 3 Predictive factors at multivariable analysis.

\begin{tabular}{llll}
\hline & Hazard ratio & \multicolumn{1}{c}{$\mathbf{9 5 \%} \mathbf{C l}$} & $\boldsymbol{P}$ \\
\hline Recurrence-free survival & & & \\
Sex & 0.774 & $0.387-1.547$ & 0.468 \\
Age at diagnosis & 1.001 & $0.977-1.027$ & 0.909 \\
Secreting tumor & 1.864 & $0.93-3.75$ & 0.081 \\
Tumor stage & 1.728 & $0.862-3.464$ & 0.123 \\
Weiss score & 1.145 & $0.909-1.443$ & 0.250 \\
High mitotic index & 2.112 & $0.97-4.47$ & 0.051 \\
Maintenance of mitotane & 0.418 & $0.220-0.791$ & 0.007 \\
$\quad$ levels $\geq 14$ mg/l & & & \\
Overall survival & & & \\
Sex & 1.418 & $0.553-3.636$ & 0.468 \\
Age at diagnosis & 1.029 & $0.995-1.065$ & 0.097 \\
Secreting tumor & 2.31 & $0.80-6.70$ & 0.122 \\
$\quad$ Tumor stage & 1.725 & $0.732-4.064$ & 0.212 \\
Weiss score & 1.088 & $0.823-1.438$ & 0.552 \\
High mitotic index & 2.99 & $1.06-8.50$ & 0.039 \\
$\quad$ Maintenance of mitotane & 0.588 & $0.257-1.344$ & 0.208 \\
$\quad$ levels $\geq 14$ mg/l & & & \\
\hline
\end{tabular}


Table 4 Adverse events ${ }^{a}$.

\begin{tabular}{lccccc}
\hline & \multicolumn{5}{c}{ Grades } \\
\cline { 2 - 5 } Toxicity & 1 & 2 & 3 & 4 & Missing \\
\hline No. of patients (\%) & $19(15.6)$ & $7(5.7)$ & $7(5.7)$ & 0 & $8(6.5)$ \\
$\quad$ Neurologic & $23(18.8)$ & $8(6.5)$ & 0 & 0 & $6(4.9)$ \\
Hepatic & $22(18.0)$ & $22(18.0)$ & $3(2.4)$ & $1(0.8)$ & $5(3.3)$ \\
Gastro-intestinal &
\end{tabular}

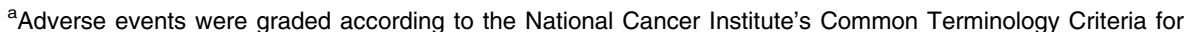
Adverse Events. Owing to the adrenolytic action of mitotane, all patients received prophylactic glucocorticoid replacement therapy. Therefore, detailed monitoring of mitotane-induced adrenal insufficiency was not performed. Because of the retrospective nature of the study, underreporting of low-grade side effects must be considered a possibility.

\section{Discussion}

More than 50 years after the introduction of mitotane in clinical practice (8), its use as a postoperative adjunctive measure remains a matter of debate $(11,12,23)$.

This study shows for the first time that mitotane levels may influence the outcome of patients treated adjuvantly following radical resection of ACC and validate the concept of a therapeutic value for a cutoff of mitotane concentrations at $14 \mathrm{mg} / \mathrm{l}$. Patients who were able to consistently maintain mitotane levels of more than $14 \mathrm{mg} / \mathrm{l}$ during follow-up had a significantly reduced risk of recurrence than patients who failed to do so. The apparent benefit of elevated mitotane concentrations was confirmed when multivariable analyses were used to adjust for the most important prognostic factors $(13,24,25)$. OS appeared to be nonsignificantly prolonged in patients who maintained target mitotane concentrations. However, since the number of events (deaths) was low $(27 \%)$ and the median survival was not yet reached, it is likely that the effect will become fully evident only after a longer duration of follow-up. Even accepting that OS is not significantly extended by adjuvant mitotane, treatment may be justified because ACC events, such as distant and loco-regional recurrences, are clinically important for patients. Although mitotane may impair the quality of life because of unwanted effects, treatment-related toxicity is sustainable; thus, extending the duration of life free of disease may benefit ACC patients as tumor recurrence has a great negative impact on the quality of life, and either psychological or physical health of affected patients, and triggers even more toxic treatments.

RFS is increasingly considered as a valid surrogate endpoint for OS in adjuvant trials being closely related to the efficacy of adjuvant treatment, whereas OS is usually also affected by the treatment adopted upon progression $(26,27)$. However, RFS strongly depends on the frequency and the quality of follow-up procedures that were comparable among the different centers.

The independent relationship of mitotane concentration with RFS observed in this study is a further step toward demonstration of the efficacy of mitotane in an adjuvant setting, although we cannot exclude that reaching target plasma concentrations is associated with unexplored patient or tumor features that per se could be predictive of a better prognosis.

The available evidence on target mitotane concentrations has been obtained only in the clinical scenario of advanced ACC; however, most experts recommend targeting the cutoff level of $14 \mathrm{mg} / \mathrm{l}$ also in the adjuvant setting $(13,17)$. The present results validate this treatment strategy, since a consistent maintenance of mitotane target concentrations during treatment was an independent factor associated with a lower risk of recurrence. This finding is likely to have a major influence on clinical practice prompting a more stringent approach at maintaining target mitotane concentrations. The present evidence should strengthen the commitment of both patients and physicians to this task, that is difficult to fulfill, as shown in this study. As levels of $14 \mathrm{mg} / \mathrm{l}$ have been targeted in our patients to guide mitotane dosing, we did not analyze other cutoff values that were not used in the clinical decision process.

There is currently controversy on the best dosing regimen to initiate mitotane therapy. In our study, either high-dose or low-dose regimens were employed reflecting this controversy. A high-dose regimen has been advocated to allow a rapid rise of plasma mitotane concentrations in order to avoid any delay in drug activity $(28,29)$; however, toxicity associated with such a regimen is of concern. On the other hand, also a lowdose regimen, potentially better tolerated, is able to provide target mitotane concentrations, but with a time lag of several months from treatment start (30). It is noteworthy that in the present series the percentage of patients attaining target mitotane concentrations at the landmark of 3 months did not differ in the high-dose vs low-dose group. Furthermore, the time elapsed to attain target mitotane concentrations did not seem to add prognostic information, suggesting that shortening of this period, although plausible, is not of paramount importance. However, as this was not a pharmacokinetic study we cannot finally answer the question about the best treatment regimen. 
Toxicity was overall acceptable and it was possible to manage unwanted effects with temporary treatment discontinuation, or dose reduction, without losing any patient for compliance-related issues. Despite a possible underreporting of some adverse effects due to the retrospective nature of the study, we confirm that adjuvant mitotane treatment is feasible proven that a careful follow-up and counseling is offered to the patients. The monitoring of mitotane concentrations is of pivotal importance in this context to guide dose adjustments and limit unwanted effects, as it was previously observed (30). Conversely, studies published before the availability of mitotane monitoring reported that a severe and disabling toxicity was associated with mitotane use, which may lead many patients to be unable to perform the usual activities of ordinary life (7).

Our study had certain limitations, the retrospective nature and absence of randomization are the most important ones. To reduce selection bias that was mainly towards patients at perceived high risk of recurrence, we included all consecutive patients who were given adjuvant mitotane at the participating centers. Differences in histological classification among the centers are unlikely, since all tumor specimens were reviewed by expert pathologists who used the same classification criteria $(18,19)$. Diagnostic and staging protocols were similar in all centers, and patients underwent surgery during the same period. Finally, follow-up procedures were largely similar among the centers and complete follow-up data were available for all patients. Thus, as far as can be stated in a retrospective study, biases appear to have been minimal.

In conclusion, this is the first demonstration that plasma mitotane concentrations may have a therapeutic impact in an adjuvant setting, as mitotane levels of $14 \mathrm{mg} / \mathrm{l}$, or higher, may prolong RFS in patients with radically resected ACC. This finding supports indirectly the efficacy of adjuvant mitotane treatment and adds to recent evidence of a beneficial effect of mitotane in an adjuvant setting $(31,32)$. Additional findings of our study that may have an impact on practice are that mitotane concentrations can be used as a factor predicting the response to treatment, and that the dosing schedule used at the initiation of mitotane treatment is less important than striving for reaching and maintaining target mitotane levels during adjuvant therapy. This piece of evidence may help in structuring an articulated systemic treatment of ACC that remains challenging as the rarity of the disease hampered the development of effective therapeutic options beyond surgery (33).

\section{Declaration of interest}

M Terzolo, A E Baudin, H R Haak, B Allolio, and M Fassnacht participated in a study on the pharmacokinetics of mitotane in a subgroup of patients who were enrolled in the FIRM-ACT study. This sub-study was funded by HRA Pharma.

\section{Funding}

This study was supported by grants of the Italian Ministry of University and Scientific and Technological Research (PRIN, grant no. 20085P5S49 to M Terzolo), Deutsche Krebshilfe (\#106 080 to B Allolio and M Fassnacht, and \#107 111 to M Fassnacht), Regione Piemonte (Ricerca Sanitaria Finalizzata 2006 to M Volante and Ricerca Sanitaria Finalizzata 2008 to A Berruti). The study was done under the auspices of the European Network for the Study of Adrenal Tumors (ENS@T).

\section{References}

1 Roman S. Adrenocortical carcinoma. Current Opinion in Oncology 200618 36-42. (doi:10.1097/01.cco.0000198976.43992.14)

2 Terzolo M, Alì A, Osella G \& Mazza E. Prevalence of adrenal carcinoma among incidentally discovered adrenal masses. A retrospective study from 1989 to 1994. Archives of Surgery 1997 132 914-919. (doi:10.1001/archsurg.1997.01430320116020)

3 Schteingart DE, Doherty GM, Gauger PG, Giordano TJ, Hammer GD, Korobkin M \& Worden FP. Management of patients with adrenal cancer: recommendations of an International Consensus Conference. Endocrine-Related Cancer 200512 667-680. (doi:10.1677/erc.1.01029)

4 Allolio B \& Fassnacht M. Adrenocortical carcinoma: clinical update. Journal of Clinical Endocrinology and Metabolism 200691 2027-2037. (doi:10.1210/jc.2005-2639)

5 Bilimoria KY, Shen WT, Elaraj D, Bentrem DJ, Winchester DJ, Kebebew E \& Sturgeon C. Adrenocortical carcinoma in the United States: treatment utilization and prognostic factors. Cancer 2008 113 3130-3136. (doi:10.1002/cncr.23886)

6 Icard P, Goudet P, Charpenay C, Andreassian B, Carnaille B, Chapuis Y, Cougard P, Henry JF \& Proye C. Adrenocortical carcinomas: surgical trends and results of a 253-patient series from the French Association of Endocrine Surgeons study group. World Journal of Surgery 200125 891-897. (doi:10.1007/ s00268-001-0047-y)

7 Schulick RD \& Brennan MF. Long-term survival after complete resection and repeat resection in patients with adrenocortical carcinoma. Annals of Surgical Oncology $19996719-726$. (doi:10.1007/s10434-999-0719-7)

8 Bergenstal DM, Lipsett MB, Moy RH \& Hertz R. Regression of adrenal cancer and suppression of adrenal function in men by o,p'-DDD. Transactions of the Association of American Physicians 195972 341-347.

9 Terzolo M, Angeli A, Fassnacht M, Daffara F, Tauchmanova L, Conton PA, Rossetto R, Buci L, Sperone P, Grossrubatscher E et al. Adjuvant mitotane treatment for adrenocortical carcinoma. New England Journal of Medicine 2007356 2372-2380. (doi:10.1056/ NEJMoa063360)

10 Schteingart DE. Adjuvant mitotane therapy of adrenal cancer use and controversy. New England Journal of Medicine $2007 \mathbf{3 5 6}$ 2415-2418. (doi:10.1056/NEJMe078087)

11 Huang $\mathrm{H} \&$ Fojo T. Adjuvant mitotane for adrenocortical cancer a recurring controversy. Journal of Clinical Endocrinology and Metabolism 2008 93 3730-3732. (doi:10.1210/jc.2008-0579)

12 Terzolo M, Fassnacht M, Ciccone G, Allolio B \& Berruti A. Adjuvant mitotane for adrenocortical cancer - working through uncertainty. Journal of Clinical Endocrinology and Metabolism 2009 94 1879-1880. (doi:10.1210/jc.2009-0120)

13 Fassnacht M, Libé R, Kroiss M \& Allolio B. Adrenocortical carcinoma: a clinician's update. Nature Reviews. Endocrinology 20117 323-335. (doi:10.1038/nrendo.2010.235)

14 Haak HR, Hermans J, van de Velde CJ, Lentjes EG, Goslings BM, Fleuren GJ \& Krans HM. Optimal treatment of adrenocortical carcinoma with mitotane: results in a consecutive series of 96 patients. British Journal of Cancer 199469 947-951. (doi:10.1038/bjc.1994.183) 
15 Baudin E, Pellegriti G, Bonnay M, Penfornis A, Laplanche A, Vassal $G$ \& Schlumberger $M$. Impact of monitoring plasma 1,1-dichlorodiphenildichloroethane (o,p'DDD) levels on the treatment of patients with adrenocortical carcinoma. Cancer 200192 1385-1392. (doi:10.1002/1097-0142(20010915)92:6<1385 $:: A I D-C N C R 1461>3.0 . C O ; 2-2)$

16 Hermsen IG, Fassnacht M, Terzolo M, Houterman S, den Hartigh J, Leboulleux S, Daffara F, Berruti A, Chadarevian R, Schlumberger $\mathrm{M}$ et al. Plasma concentrations of o,p'DDD, $o, p$ 'DDA, and o,p'DDE as predictors of tumor response to mitotane in adrenocortical carcinoma: results of a retrospective ENS@T multicenter study. Journal of Clinical Endocrinology and Metabolism 201196 1844-1851. (doi:10.1210/jc.2010-2676)

17 Terzolo M \& Berruti A. Adjunctive treatment of adrenocortical carcinoma. Current Opinion in Endocrinology, Diabetes, and Obesity 200815 221-226. (doi:10.1097/MED.0b013e3282 fdf $4 \mathrm{c} 0$ )

18 Weiss LM. Comparative histologic study of 43 metastasizing and nonmetastasizing adrenocortical tumors. American Journal of Surgical Pathology 1984 8 163-169. (doi:10.1097/00000478198403000-00001)

19 Weiss LM, Medeiros LJ \& Vickery AL Jr. Pathologic features of prognostic significance in adrenocortical carcinoma. American Journal of Surgical Pathology 198913 202-206. (doi:10.1097/ 00000478-198903000-00004)

20 Fassnacht M, Johanssen S, Quinkler M, Bucsky P, Willenberg HS, Beuschlein F, Terzolo M, Mueller HH, Hahner S, Allolio B et al. Limited prognostic value of the 2004 International Union Against Cancer staging classification for adrenocortical carcinoma: proposal for a revised TNM classification. Cancer $2009 \mathbf{1 1 5}$ 243-250. (doi:10.1002/cncr.24030)

21 Common Terminology criteria for adverse event V3.0 (CTCAE). Bethesda, MD: National Cancer Institute, 2003. Available http://ctep. cancer.gov/protocolDevelopment/electronic_applications/ctc.htm.

22 Volante M, Bollito E, Sperone P, Tavaglione V, Daffara F, Porpiglia F, Terzolo M, Berruti A \& Papotti M. Clinicopathological study of a series of 92 adrenocortical carcinomas: from a proposal of simplified diagnostic algorithm to prognostic stratification. Histopathology 200955 535-543. (doi:10.1111/j.1365-2559. 2009.03423.x)

23 Veytsman I, Nieman L \& Fojo T. Management of endocrine manifestations and the use of mitotane as a chemotherapeutic agent for adrenocortical carcinoma. Journal of Clinical Oncology 200920 4619-4629. (doi:10.1200/JCO.2008.17.2775)

24 Malandrino P, Al Ghuzlan A, Castaing M, Young J, Caillou B, Travagli JP, Elias D, de Baere T, Dromain C, Paci A et al. Prognostic markers of survival after combined mitotane- and platinum-based chemotherapy in metastatic adrenocortical carcinoma. EndocrineRelated Cancer 201017 797-807. (doi:10.1677/ERC-09-0341)

25 Baudin E, Leboulleux S, Al Ghuzlan A, Chougnet C, Young J, Deandreis D, Dumont F, Dechamps F, Caramella C, Chanson P et al.
Therapeutic management of advanced adrenocortical carcinoma: what do we know in 2011? Hormones \& Cancer 20112 363-371. (doi:10.1007/s12672-011-0094-2)

26 Burstein HJ, Prestrud AA, Seidenfeld J, Anderson H, Buchholz TA, Davidson NE, Gelmon KE, Giordano SH, Hudis CA, Malin J et al. American Society of Clinical Oncology clinical practice guideline: update on adjuvant endocrine therapy for women with hormone receptor-positive breast cancer. Journal of Clinical Oncology 2010 28 3784-3796. (doi:10.1200/JCO.2009.26.3756)

27 Sargent DJ, Wieand HS, Haller DG, Gray R, Benedetti JK, Buyse M, Labianca R, Seitz JF, O'Callaghan CJ, Francini G et al. Disease-free survival vs overall survival as a primary end point for adjuvant colon cancer studies: individual patient data from 20,898 patients on 18 randomized trials. Journal of Clinical Oncology 200523 8664-8670. (doi:10.1200/JC0.2005.01.6071)

28 Faggiano A, Leboulleux S, Young J, Schlumberger M \& Baudin E. Rapidly progressing high o,p'DDD doses shorten the time required to reach the therapeutic threshold with an acceptable tolerance: preliminary results. Clinical Endocrinology 200664 110-113. (doi:10.1111/j.1365-2265.2005.02403.x)

29 Mauclère-Denost S, Leboulleux S, Borget I, Paci A, Young J, Al Ghuzlan A, Deandreis D, Drouard L, Tabarin A, Chanson P et al. High-dose mitotane strategy in adrenocortical carcinoma: prospective analysis of plasma mitotane measurement during the first 3 months of follow-up. European Journal of Endocrinology 2012 166 261-268. (doi:10.1530/EJE-11-0557)

30 Daffara F, De Francia S, Reimondo G, Zaggia B, Aroasio E, Porpiglia F, Volante M, Termine A, Di Carlo F, Dogliotti L et al. Prospective evaluation of mitotane toxicity in adrenocortical cancer patients treated adjuvantly. Endocrine-Related Cancer 2008 15 1043-1053. (doi:10.1677/ERC-08-0103)

31 Wängberg B, Khorram-Manesh A, Jansson S, Nilsson B, Nilsson O, Jakobsson CE, Lindstedt S, Odén A \& Ahlman H. The longterm survival in adrenocortical carcinoma with active surgical management and use of monitored mitotane. Endocrine-Related Cancer 201017 265-272. (doi:10.1677/ERC-09-0190)

32 Fassnacht M, Johanssen S, Fenske W, Weismann D, Agha A, Beuschlein F, Führer D, Jurowich C, Quinkler M, Petersenn S et al. Improved survival in patients with stage II adrenocortical carcinoma followed up prospectively by specialized centers. Journal of Clinical Endocrinology and Metabolism 201095 4925-4932. (doi:10.1210/jc.2010-0803)

33 Fassnacht M, Terzolo M, Allolio B, Baudin E, Haak H, Berruti A, Welin S, Schade-Brittinger C, Lacroix A, Jarzab B et al. Combination chemotherapy in advanced adrenocortical carcinoma. New England Journal of Medicine 2012366 2189-2197. (doi:10.1056/NEJMoa1200966)

Received 23 March 2013

Revised version received 19 May 2013

Accepted 23 May 2013 